\title{
Utilização de redes neurais artificiais na classificação de níveis de degradação em pastagens
}

\author{
César S. Chagas ${ }^{1}$, Carlos A. O. Vieira ${ }^{2}$, Elpídio I. Fernandes Filho ${ }^{3} \&$ Waldir de C. Júnior ${ }^{4}$
}

\begin{abstract}
RESUMO
Este trabalho teve por objetivo avaliar a eficiência dos classificadores redes neurais artificiais (RNA) e o de máxima verossimilhança (Maxver) na classificação do uso da terra no município de Viçosa, MG, a partir de imagens do sensor ASTER, com ênfase nos níveis de degradação das pastagens. Neste estudo, foram identificados três níveis de degradação das pastagens (moderado, forte e muito forte) e avaliada uma composição da imagem do sensor ASTER contendo as 3 bandas do visível e infravermelho próximo, com resolução espacial de $15 \mathrm{~m}$. O simulador de redes neurais empregado foi o "Java Neural Network Simulator" e o algoritmo de aprendizado, o backpropagation. Os resultados mostram que a classificação por redes neurais, embora apresente resultado ligeiramente superior, teve desempenho estatisticamente semelhante ao obtido pela classificação pelo Maxver, obtendo um índice Kappa de 0,80, contra 0,79, respectivamente. Nas classificações realizadas a classe que apresentou maior erro de classificação foi a pastagem no nível de degradação forte, enquanto a maior exatidão na classificação foi obtida pelo café, para ambos os classificadores, com 100 e 96\%, respectivamente.
\end{abstract}

Palavras-chave: aster, sensoriamento remoto, classificação supervisionada

\section{Use of artificial neural networks in the classification of degradation levels of pastures}

\begin{abstract}
The aim of this work is to evaluate the artificial neural networks and the maximum likelihood classification performances to classify land uses at Viçosa, Minas Gerais State, using ASTER images in order to verify degradation levels of pastures. In this study, three different levels of pasture degradation have been identified (moderate, strong and very strong) and an image composition of 3 bands was tested (covering the visible and the near infra-red) with $15 \mathrm{~m}$ of spatial resolution. The neural networks simulator used was the "Neural Java Network Simulator", with a feed forward model and the learning algorithm of back propagation. The obtained results show that the classification using neural networks, while presenting a slightly superior result, had a statistically similar performance compared to the maximum likelihood, getting a Kappa index of 0.80 , against 0.79 , respectively. In relation to individual performances, the class that presented the greatest error of classification was pasture in the level of very strong degradation, while the largest accuracy in the classification was obtained for coffee, for both classifiers, with 100 and 96\% (respectively, Maxver and neural networks).
\end{abstract}

Key words: aster, remote sensing, supervised classification

1 Embrapa Solos. Rua Jardim Botânico 1024, Jardim Botânico, CEP 22460-000, Rio de Janeiro, RJ. Fone (21) 2179-4546. E-mail: chagas.rj@gmail.com 2 DEC/UFV, CEP 36570000, Viçosa, MG. Fone (31)3899-3036. E-mail: carlos.vieira@ufv.br

3 DPS/UFV. Fone (31) 3899-1040. E-mail: elpidio@ufv.br

${ }^{4}$ Embrapa Solos. Fone (21) 2179-4582. E-mail: waldircj@cnps.embrapa.br 


\section{INTRODUÇÃO}

Verifica-se, na Zona da Mata Mineira, um predomínio quase total da utilização das terras com pastagens. O manejo inadequado e as deficiências nutricionais do solo são os fatores que mais concorrem para a degradação das pastagens, nesta região (Carvalho, 1998).

A análise de dados de sensores remotos, devido ao seu baixo custo, periodicidade e escala compatível, pode contribuir muito para a identificação de níveis de degradação em pastagens (Sano et al., 2000); no entanto, as tentativas para mapear pastagens em regiões tropicais e subtropicais freqüentemente têm sucesso limitado, devido a grande confusão com outras feições.

Várias técnicas vêm sendo aplicadas na análise e classificação do uso da terra, através de dados de sensoriamento remoto. Neste sentido, vários autores têm ressaltado que diferentes algoritmos de classificação produzem diferentes resultados, até mesmo quando se utiliza o mesmo conjunto de amostras de treinamento (Benediktsson et al., 1990; Hepner et al., 1990; Bischof et al., 1992; Kanellopoulos et al., 1992; Civco, 1993; Skidmore et al., 1997).

As aplicações do algoritmo de máxima verossimilhança (Maxver) são bastante conhecidas na literatura de sensoriamento remoto, estando este algoritmo presente em vários softwares de processamento de imagens; e frequentemente, é usado como rotina de classificação padrão contra o qual outros algoritmos de classificação são comparados. Este algoritmo identifica a forma, o tamanho e a orientação das amostras de treinamento (Vieira, 2000). Se a hipótese assumida de uma distribuição normal para cada classe da área de treinamento está correta, então a classificação tem uma probabilidade mínima de erro e o algoritmo é uma escolha acertada (Paola \& Schowengerdt, 1994). Neste sentido, Nascimento et al. (2006) utilizaram imagens do sensor ASTER na identificação de níveis de degradação em pastagens, na região de Viçosa, MG, usando o algoritmo de classificação distância de Mahalanobis (que possui formulação similar ao classificador Maxver). A exatidão global obtida para a classificação realizada foi de $85,07 \%$ e o índice Kappa de 0,83, usando-se as bandas do visível e infravermelho próximo que possuem resolução espacial de $15 \mathrm{~m}$.

Na última década, as redes neurais artificiais têm experimentado um grande interesse e têm sido aplicadas com sucesso no reconhecimento de padrões de imagens de satélite. Uma das principais vantagens das redes neurais é a possibilidade de manipulação eficiente de grandes quantidades de dados, mas a principal razão para o seu uso na classificação de dados de sensoriamento remoto e dados geográficos, é que as redes neurais não assumem nenhuma distribuição, diferentemente da estatística paramétrica tradicional, que pressupõe que as classes informacionais apresentam distribuição normal (Atkinson \& Tatnall, 1997).

Segundo Yool (1998), os resultados alcançados até o momento sugerem que as redes neurais podem ser robustas quando dados espectrais são indistintos ou esparsos, e que as redes são capazes de produzir exatidões que excedem a maioria dos métodos de reconhecimento de padrão que utilizam a estatística convencional.

Hepner et al. (1990) utilizaram redes neurais para classificar a cobertura do terreno a partir de imagens do Landsat TM e concluíram que abordagem de redes neurais foi mais eficiente que a classificação estatística tradicional. Bischof et al. (1992) utilizaram uma rede neural para classificar imagens multiespectrais e compararam os resultados com aqueles obtidos pelo classificador Maxver. Esses autores relataram, ainda, uma exatidão global de 93,1\% contra 89,7\% obtidos com o uso do Maxver. Venkatesh \& Raja (2003) também utilizaram redes neurais artificiais na classificação de imagens de satélites multiespectrais. As classes de cobertura identificadas foram: florestas, agricultura, montanhas, estradas, terrenos rochosos e corpos d’água. A abordagem empregada resultou em uma exatidão de mais de $99 \%$ na discriminação das classes citadas.

Embora se tenha utilizado em vários estudos, redes neurais na classificação de diversas classes informacionais a partir de dados de sensores remotos, o presente estudo se destaca como uma primeira tentativa na classificação de uma mesma classe informacional (pastagem) em diferentes níveis de degradação, a partir de dados do sensor ASTER empregando-se a abordagem por redes neurais; assim, este estudo tem como objetivo comparar a eficiência de redes neurais artificiais com o classificador Maxver para a classificação do uso das terras, com ênfase nos níveis de degradação das pastagens, em Viçosa, Minas Gerais.

\section{MATERIAL E MÉTODOS}

\section{Área de estudo}

A área estudada compreende a bacia do ribeirão São Bartolomeu, no Município de Viçosa, Minas Gerais, localizada entre as coordenadas UTM 7706390 e 7694049 m N e 716754 e 724428 m E, fuso 23S (Figura 1). O ribeirão São Bartolomeu é responsável por parte do abastecimento de água da cidade de Viçosa e parte da água utilizada na Universidade Federal de Viçosa. Assim como toda a região da Zona da Mata de Minas Gerais, a área da bacia é caracterizada por uma topografia bastante acidentada. São encontrados, ainda, na bacia, vales cujos fundos correspondem ao leito maior, periodicamente inundável, seguido de terraços assimétricos onde é mais freqüente a prática de agricultura e habitações (Vilela, 1998). Os processos de expansão urbana e de implantação de pastagens e, em menor escala, cultivos agrícolas, provocaram a substituição de grande parte da vegetação nativa que, atualmente, é constituída de vegetação secundária localizada, de maneira dispersa nos topos de morros.

\section{Material}

Foi utilizada, neste estudo, uma imagem do sensor ASTER (Advanced Spaceborne Thermal Emission and Reflection Radiometer), obtida em 05/04/2001, que coincide com o início da estação seca na região. As bandas usadas foram as do visível e infravermelho próximo (3) com resolução espacial de $15 \mathrm{~m}$. 


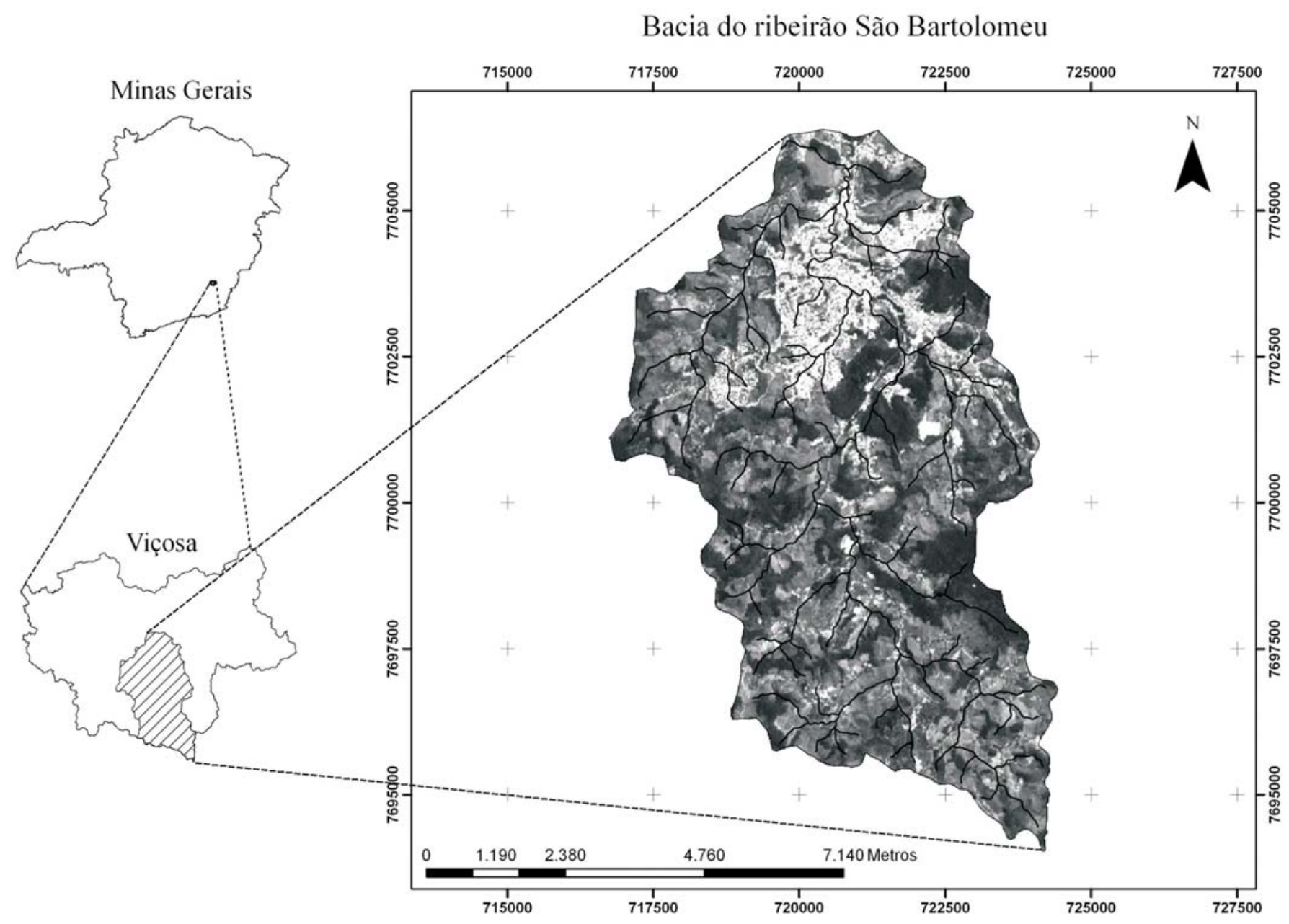

Figura 1. Localização da área estudada

O simulador de redes neurais empregado foi o "Java Neural Network Simulator” desenvolvido pelo Instituto Wilhem-Schickard para Ciência da Computação (WSI) em Tübingen, Alemanha. Este simulador é baseado no Stuttgart Neural Network Simulator 4.2 Kernel (Zell et al., 1996), com uma nova interface gráfica. O algoritmo de aprendizado foi o backpropagation desenvolvido nos anos 80, que é um algoritmo usado para classificação supervisionada.

Nas etapas de coleta de amostras de treinamento e validação, preparação dos dados e geração dos arquivos utilizados na classificação da imagem pelos dois classificadores e preparação dos dados após a classificação da imagem propriamente dita, foram utilizados os seguintes executáveis desenvolvidos por Vieira (2000): Funcpow e Gerapat; além desses, se utilizaram o software de SIG ArcGIS 9.0 e o software de processamento de imagens digitais ERDAS Imagine 8.5.

Levando-se em consideração as características das pastagens da região da Zona da Mata de Minas Gerais, com predominância da espécie Melinis minutiflora, Beauv, e os indicadores visuais de degradação, foram considerados os níveis de degradação identificados por Nascimento et al. (2006) que são: pastagem no nível de degradação moderado (pastagem 2), pastagem no nível de degradação forte (pastagem 3) e pastagem no nível de degradação muito forte (pastagem 4), conforme apresentado na Tabela 1.

Tabela 1. Níveis de degradação de pastagens utilizados

\begin{tabular}{|c|c|c|}
\hline $\begin{array}{l}\text { Níveis de } \\
\text { degradação }\end{array}$ & Parâmetros limitantes & Deterioração \\
\hline Pastagem 1 & Bom vigor e boa qualidade & Leve (não identificado) \\
\hline Pastagem 2 & Baixo vigor, qualidade e baixa população & Moderado \\
\hline Pastagem 3 & $\begin{array}{l}\text { Baixo vigor, qualidade e baixa população, } \\
\text { associado com a presença de invasoras } \\
\text { e/ou cupins }\end{array}$ & Forte \\
\hline Pastagem 4 & $\begin{array}{l}\text { Baixo vigor, qualidade e baixa população, } \\
\text { associado com a presença de invasoras, } \\
\text { cupins e solo descoberto }\end{array}$ & Muito forte \\
\hline
\end{tabular}

Fonte: Adaptado de Spain \& Gualdrón (1988) e Moreira \& Assad (2000)

\section{Metodologia}

A classificação da imagem pelos dois classificadores foi realizada com os mesmos conjuntos de dados; assim, foram coletados dois conjuntos de amostras independentes, um para treinamento e outro para validação. O número de amostras para treinamento foi estipulado em 250 para cada classe e o tamanho do conjunto de validação em 150; além das classes referentes aos níveis de degradação das pasta- 
gens, descritas anteriormente, consideraram-se também as classes mata (floresta primária ou secundária), café e área urbana/solo exposto, totalizando 6 classes informacionais. As curvas de respostas espectrais médias dos conjuntos de dados de treinamento dessas classes são mostradas na Figura 2.

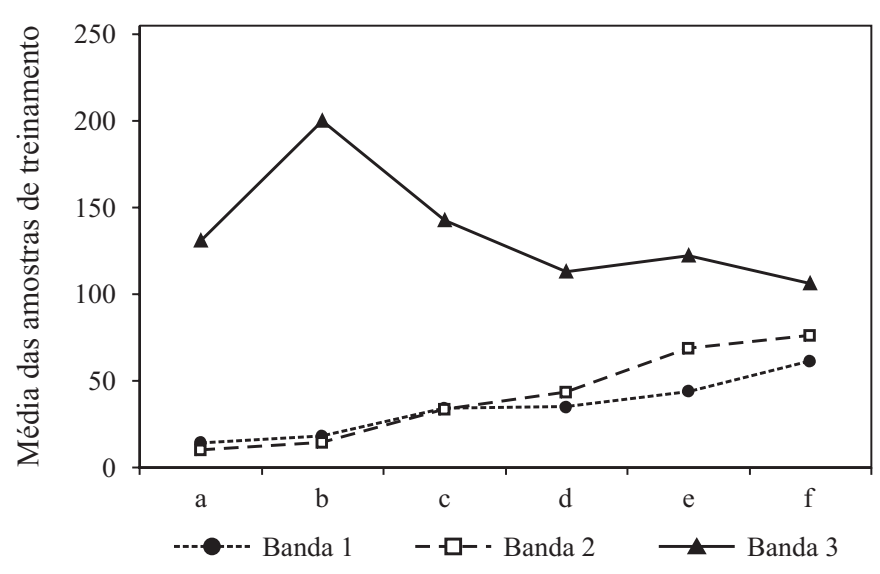

Figura 2. Distribuição das variáveis discriminantes entre as classes avaliadas. $\mathrm{a}$ - mata; $\mathrm{b}$ - café; c - pastagem 2 ; $\mathrm{d}$ - pastagem 3; e - pastagem 4 e f área urbana/solo exposto

Segundo Vieira (2000), a seleção do conjunto de dados de treinamento requer algum cuidado. Alguns pesquisadores consideram que um conjunto selecionado aleatoriamente é a melhor opção; entretanto, o conjunto ideal de treinamento não é aleatório, mas aquele no qual cada exemplo pode ser usado pelo modelo para reforçar um princípio diferente da relação de entrada-saída, assim, a amostragem utilizada foi a do tipo aleatória estratificada. Para tanto, e com base nos padrões das classes informacionais obtidos no campo com GPS (Ground Position System), foram coletadas, aleatoriamente, amostras dentro de cada uma das classes consideradas.

Uma vez definidos os conjuntos de amostras contendo as informações relativas à imagem utilizada, todos os dados da imagem do sensor ASTER foram reescalonados entre os valores de 0 e 1 , para facilitar o processo de treinamento da rede e simplificar sua estrutura; isto foi feito para evitar a saturação da rede, primeiro porque valores grandes poderiam impedir a solução do problema e, segundo, para prevenir que grandes variações de uma variável pouco importante escondam pequenas, porém importantes variações em outras variáveis. Embora não existam limites teóricos sobre os valores de entrada das redes neurais, sua estabilidade é usualmente melhorada quando se usam limites comparáveis entre variáveis (Master, 1993).

Realizaram-se, após este procedimento, o treinamento e a validação das amostras pelo classificador Maxver. Esta etapa foi implementada no executável denominado "Funcpow” desenvolvido por Vieira (2000). A opção pelo emprego deste executável se deve à possibilidade de utilização dos mesmos formatos de arquivos de treinamento e validação que foram usados para a classificação pelas redes neurais; em seguida, esses mesmos conjuntos de amostras foram utilizados no treinamento e validação das redes neurais. Diferen- tes arquiteturas foram testadas, todas com o mesmo número de neurônios na camada de entrada (3 bandas do ASTER), variando o número de neurônios na camada interna e/ou números de camadas internas e todas tendo o mesmo número de neurônios na camada de saída (6 classes de uso terra).

O número de neurônios da camada(s) interna(s) foi determinado através de tentativa e erro, conforme procedimento estabelecido por Hirose et al. (1991). O erro do treinamento mede a diferença entre os valores estimados e os valores desejados para o conjunto de treinamento.

O treinamento das redes utilizando-se um aprendizado supervisionado, consistiu dos seguintes procedimentos:

i) alocação aleatória dos pesos interneurônios. Os pesos foram inicializados com pequenos valores aleatórios (entre -0,5 e 0,5$)$ para evitar o problema de que a rede neural seja saturada com valores grandes ou a dificuldade da rede em aprender devido à presença de valores homogêneos (Vieira, 2000);

ii) determinação da taxa de aprendizado. Esta determinação para um problema específico não é tarefa simples. Valores típicos de $\eta$ estão na faixa de 0,1 a 1,0. Poucos são os exemplos em que o treinamento é mais rápido com valores acima de 1,0; algumas vezes, o valor de $\eta$ apropriado para começar o treinamento é inapropriado quando uma rede alcança pontos mais próximos ao seu mínimo global, ao término do treinamento (Cauldill, 1991). A taxa de aprendizado utilizada foi de 0,2, considerando-se 10.000 ciclos de aprendizagem.

Ao final de todo o processo de treinamento, as redes que apresentaram os menores valores de MSE (Mean Square Error, erro médio quadrático) foram submetidas a validação para escolha daquela(s) que seria(m) utilizada(s) na classificação do uso da terra da área estudada em comparação com a imagem classificada pelo algoritmo Maxver.

A avaliação constou da determinação do nível de exatidão ou confiança da classificação (índice Kappa) e da exatidão global da classificação de cada rede considerada e do algoritmo Maxver, através da análise da matriz de confusão (Congalton, 1991). A matriz de confusão tem sido reconhecida como a mais importante técnica de estimativa da exatidão de produtos de dados de sensores remotos (Story \& Congalton, 1986; Skidmore \& Turner, 1989).

A exatidão global $\left(\mathrm{P}_{0}\right)$ foi computada pela divisão da soma da diagonal principal (número de classificações corretas ou concordância real) pelo número total de amostras tomadas, segundo a Equação:

$$
\mathrm{P}_{\mathrm{o}}=\frac{\sum_{\mathrm{i}=1}^{\mathrm{m}} \mathrm{n}_{\mathrm{ii}}}{\mathrm{N}}
$$

donde $\mathrm{N}$ representa o número total de amostras contempladas pela matriz de confusão; $\mathrm{n}_{\mathrm{ii}}$ representa os elementos da diagonal principal e m, o número de classes presentes na matriz.

O Coeficiente Kappa foi obtido segundo a Equação:

$$
\mathrm{Ka}=\frac{\mathrm{n} \sum_{\mathrm{i}=1}^{\mathrm{c}} \mathrm{x}_{\mathrm{ii}}-\sum_{\mathrm{i}=1}^{\mathrm{c}} \mathrm{x}_{\mathrm{i} \oplus} \mathrm{x}_{\mathrm{i} \oplus \mathrm{i}}}{\mathrm{n}^{2}-\sum_{\mathrm{i}=1}^{\mathrm{c}} \mathrm{x}_{\mathrm{i} \oplus} \mathrm{x}_{\oplus \mathrm{i}}}
$$


donde Ka é uma estimativa do coeficiente Kappa; $\mathrm{x}_{\mathrm{ii}}$ é o valor na linha i e coluna $\mathrm{i}$; linhas totais $\mathrm{x}_{\mathrm{i} \oplus}$ é a soma de linhas i e colunas totais $\mathrm{x}_{\oplus}$ é a soma das colunas i da matriz de confusão; n é o número total de amostras e c, o número total de classes.

Por sua vez, o teste estatístico para testar a significância de uma matriz de confusão individual é dado pela Equação:

$$
\mathrm{Z}=\frac{\mathrm{Ka}}{\sqrt{\operatorname{var}(\mathrm{Ka})}}
$$

Z é padronizado e distribuído normalmente e var é variância do coeficiente de Kappa ( $K a)$, que pode ser computada usandose o método Delta (Bishop et al., 1975; Kalkhan et al., 1997).

Uma matriz de significância de Kappa foi gerada para verificar a existência de diferenças significativas ou não, entre os resultados obtidos pelas diferentes redes neurais testadas, de modo a permitir a escolha da melhor arquitetura de rede para a classificação final da imagem; além disso, outra matriz de significância de Kappa foi gerada para testar a significância das diferenças entre dois classificadores, conforme Congalton \& Mead (1986).

Um teste estatístico para verificar a significância das diferenças entre dois ou mais classificadores (representado pelas suas respectivas matrizes de confusão) usando-se a análise do coeficiente de Kappa é proposta como uma abordagem alternativa para comparação de matrizes de confusão independentes; desta forma, os resultados da análise de Kappa podem ser sumarizados na forma de uma matriz de significância na qual os elementos da diagonal principal indicam se o resultado da respectiva classificação é estatisticamente diferente de uma classificação randômica. Na matriz de significância os valores de $Z$ podem ser computados usando-se a Equação 3 e a variância do coeficiente Kappa obtida através da Equação 4.

$\operatorname{Var}\left(\mathrm{K}_{\mathrm{a}}\right)=\frac{1}{\mathrm{n}}\left[\frac{\theta_{1}\left(1-\theta_{1}\right)}{\left(1-\theta_{2}\right)^{2}}+\frac{2\left(1-\theta_{1}\right)\left(2 \theta_{1} \theta_{2}-\theta_{3}\right)}{\left(1-\theta_{2}\right)^{3}}+\frac{\left(1-\theta_{1}\right)^{2}\left(\theta_{4}-4_{2}^{2}\right)}{\left(1-\theta_{2}\right)^{4}}\right]$

donde:

$$
\begin{aligned}
& \theta_{1}=\frac{1}{\mathrm{n}} \sum_{\mathrm{i}=1}^{\mathrm{c}} \mathrm{x}_{\mathrm{ii}} \\
& \theta_{2}=\frac{1}{\mathrm{n}^{2}} \sum_{\mathrm{i}=1}^{\mathrm{c}} \mathrm{x}_{\mathrm{i} \oplus} \mathrm{x}_{\oplus \mathrm{i}}
\end{aligned}
$$

$$
\begin{aligned}
& \theta_{3}=\frac{1}{n^{2}} \sum_{i=1}^{c} x_{i i}\left(x_{i \oplus}+x_{\oplus i}\right) \\
& \theta_{4}=\frac{1}{n^{3}} \sum_{i=1}^{c} \sum_{j=1}^{c} x_{i j}\left(x_{j \oplus}+x_{\oplus i}\right)^{2}
\end{aligned}
$$

Por outro lado, os elementos fora da diagonal indicam, mais uma vez, se $Z \geq Z_{\alpha / 2}$, que dois classificadores independentes são significativamente diferentes. A fórmula usada para testar a significância entre dois coeficientes Kappa independentes é dada pela equação a seguir:

$$
\mathrm{Z}=\frac{\left|\mathrm{K}_{\mathrm{a} 1}-\mathrm{K}_{\mathrm{a} 2}\right|}{\sqrt{\operatorname{var}\left(\mathrm{K}_{\mathrm{a} 1}\right)+\operatorname{var}\left(\mathrm{K}_{\mathrm{a} 2}\right)}}
$$

em que $\mathrm{K}_{\mathrm{a} 1}$ e $\mathrm{K}_{\mathrm{a} 2}$ são os dois coeficientes Kappa que estão sendo comparados (Congalton \& Mead, 1986).

\section{RESULTADOS E DISCUSSÃO}

\section{Classificação pelo Maxver}

Primeiro, fez-se a classificação das amostras da imagem ASTER considerada, utilizando-se o classificador Maxver. Os resultados desta classificação, obtidos com base nas amostras de validação, estão apresentados na Tabela 2 e mostraram uma exatidão global de 82,8\% e índice Kappa de 0,793, com variância de 0,000227 .

Nesta avaliação, a classe que apresentou o maior erro na classificação foi a classe pastagem 3 (pastagem no nível de degradação forte), com 40,7\% dos pixels que deveriam ser assinalados à esta classe, sendo assinalados a outras, principalmente à classe pastagem 4, com a qual apresentou a maior confusão (24\%), e à classe pastagem 2 (13,33\%). Esta confusão também foi verificada por Nascimento et al. (2006). Por sua vez, a maior confusão constatada para a classe pastagem 4 (pastagem no nível de degradação muito forte) foi com a classe área urbana/solo exposto, com 12,67\% dos pixels sendo assinalados erroneamente a esta última. Situação semelhante foi notada para a classe área urbana/solo exposto, que também teve $12,67 \%$ dos seus pixels assinalados er-

\begin{tabular}{|c|c|c|c|c|c|c|c|c|c|}
\hline Classes & Mata & Café & Pastagem 2 & Pastagem 3 & Pastagem 4 & $\begin{array}{c}\text { Área } \\
\text { Urbana/Solo } \\
\text { exposto }\end{array}$ & Total & $\begin{array}{l}\text { Exatidão do } \\
\text { usuário }\end{array}$ & Z \\
\hline Mata & 140 & 0 & 3 & 0 & 0 & 1 & 144 & 97,2 & 59,112 \\
\hline Café & 1 & 150 & 0 & 0 & 0 & 1 & 152 & 98,7 & 88,956 \\
\hline Pastagem 2 & 9 & 0 & 141 & 20 & 7 & 7 & 184 & 76,6 & 20,285 \\
\hline Pastagem 3 & 0 & 0 & 4 & 89 & 13 & 8 & 114 & 78,1 & 16,339 \\
\hline Pastagem 4 & 0 & 0 & 0 & 36 & 111 & 19 & 166 & 66,9 & 14,719 \\
\hline Área Urbana/solo exposto & 0 & 0 & 2 & 5 & 19 & 114 & 140 & 81,4 & 20,348 \\
\hline Total & 150 & 150 & 150 & 150 & 150 & 150 & 900 & & \\
\hline Exatidão do Produtor & 93,3 & 100,0 & 94,0 & 59,3 & 74,0 & 76,0 & & & \\
\hline
\end{tabular}
roneamente à classe pastagem 4 .

Por outro lado, mata, café e pastagem 2 (pastagem no nível de degradação moderado, respectivamente, com exatidão de 93,3, 100 e 94\% dos pixels corretamente classificados)

Tabela 2. Matriz de confusão da classificação pelo Maxver

Exatidão global $=82,8 ;$ Kappa $=0,793 ;$ Variância $=0,000227 ; Z$ calculado $=52,68$ e Z tabelado $=1,96$ 
foram as classes que apresentaram os melhores resultados, conforme a Tabela 2 .

\section{Classificação pelas Redes Neurais}

Realizou-se a classificação da imagem ASTER pelas redes neurais, com o mesmo conjunto de amostras de treinamento e validação utilizado na classificação pelo Maxver. A comparação inicial constou da verificação do MSE após 10.000 ciclos de aprendizado. Nesta avaliação foram testadas várias arquiteturas de redes para escolha daquelas que seriam submetidas a validação final, cujos resultados são mostrados na Figura 3.

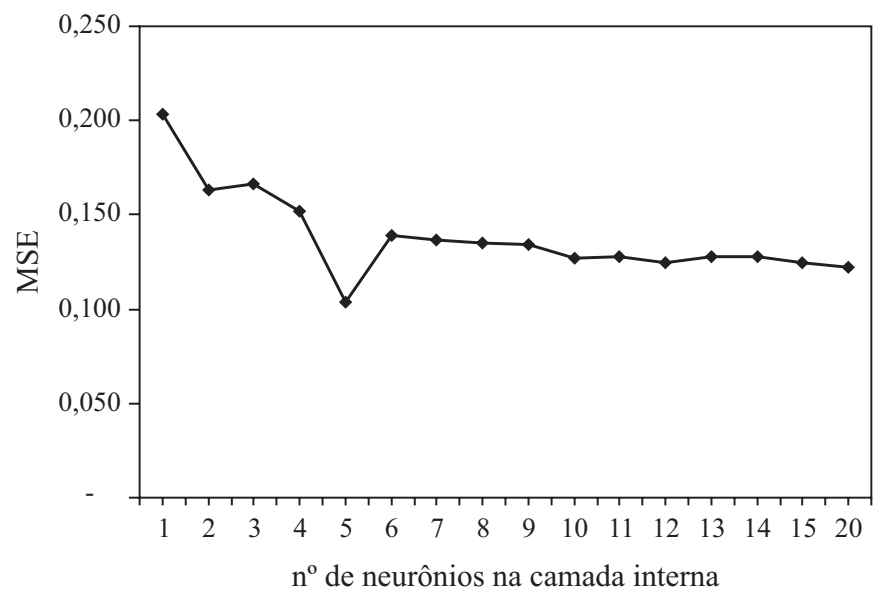

Figura 3. Resultados do treinamento das redes após 10.000 ciclos de aprendizado

O aumento da complexidade da rede com a adição de neurônios na camada interna não melhorou substancialmente o desempenho da classificação (Figura 3); desta forma, se selecionaram com base nesta avaliação, 6 arquiteturas de redes, contendo uma camada interna (5, 6, 7, 8, 9 e 10 neurônios, respectivamente), as quais foram então validadas e uma matriz de significância de Kappa foi gerada para avaliar a existência de diferenças ou não entre as redes, além de possibilitar a escolha de uma arquitetura que permitisse uma exatidão melhor da classificação (Tabela 3).

Segundo Foody \& Arora (1997), a arquitetura de uma rede neural artificial pode ter influência significativa em sua habilidade para classificar dados de sensores remotos. Nesta
Tabela 3. Matriz de significância de Kappa para as arquiteturas de redes neurais testadas

\begin{tabular}{ccccccc}
\hline Redes & $\mathbf{5}$ & $\mathbf{6}$ & $\mathbf{7}$ & $\mathbf{8}$ & $\mathbf{9}$ & $\mathbf{1 0}$ \\
Exatidão global & 82,4 & 83,3 & 79,9 & 81,3 & 82,1 & 84,2 \\
Kappa & 0,789 & 0,800 & 0,759 & 0,776 & 0,785 & 0,811 \\
Variância $^{1}$ & 0,231 & 0,222 & 0,256 & 0,243 & 0,235 & 0,212 \\
5 & 51,91 & & & & & \\
6 & 0,52 & 53,69 & & & & \\
7 & 1,36 & 1,86 & 47,44 & & & \\
8 & 0,60 & 1,11 & 0,76 & 49,78 & & \\
9 & 0,19 & 0,70 & 1,17 & 0,41 & 51,21 & \\
10 & 1,05 & 0,53 & $2,40^{*}$ & 1,64 & 1,23 & 55,70 \\
\hline
\end{tabular}

* existe diferença significativa a nível de $95 \%$

${ }^{1}$ valores multiplicados por 1000

avaliação, os maiores valores de exatidão global e índice Kappa e, conseqüentemente, os menores de variância, foram obtidos com uma arquitetura de rede com apenas uma camada interna contendo 10 neurônios, seguida da rede com 6 neurônios na camada interna.

A Tabela 3 mostra, ainda, que todos os valores da diagonal são maiores que $\mathrm{Z}$ tabelado $(1,96)$, indicando que a classificação de todas as redes é significativamente melhor que uma classificação randômica. O melhor desempenho foi obtido com a utilização da rede com uma camada interna contendo 10 neurônios ( $\mathrm{Z}$ calculado de 55,70); no entanto, esta é significativamente diferente apenas da rede com 7 neurônios na camada interna.

Uma vez que redes menores generalizam melhor, a rede com 6 neurônios, por ser mais simples, foi utilizada para classificação da imagem ASTER. A matriz de confusão obtida para esta rede neural é apresentada na Tabela 4.

Nesta avaliação, a classe que apresentou o maior erro na classificação, tal como ocorreu para a classificação pelo Maxver, foi a classe pastagem 3 (pastagem no nível de degradação forte), com $29,30 \%$ dos pixels que deveriam ser assinalados a esta classe, mas o foram a outras classes, principalmente à classe pastagem 4 , com a qual apresentou a maior confusão (17,33\%) e à classe área urbana/solo exposto (8\%). Por sua vez, a maior confusão verificada para a classe pastagem 4 (pastagem no nível de degradação muito forte) foi com a classe área urbana/solo exposto, com 15 (10\%) dos 150 pixels sendo assinalados erroneamente a esta última. Situação semelhante também foi verificada para a classe área urbana/solo exposto,

Tabela 4. Matriz de confusão da classificação pela rede neural

\begin{tabular}{|c|c|c|c|c|c|c|c|c|c|}
\hline Classes & Mata & Café & Pastagem 2 & Pastagem 3 & Pastagem 4 & $\begin{array}{c}\text { Área } \\
\text { Urbana/Solo } \\
\text { exposto }\end{array}$ & Total & $\begin{array}{l}\text { Exatidão do } \\
\text { usuário }\end{array}$ & $\mathbf{Z}$ \\
\hline Mata & 139 & 4 & 1 & 0 & 0 & 0 & 144 & 96,5 & 52,668 \\
\hline Café & 1 & 144 & 6 & 0 & 0 & 3 & 154 & 93,5 & 39,177 \\
\hline Pastagem 2 & 10 & 1 & 118 & 6 & 6 & 1 & 142 & 83,1 & 21,759 \\
\hline Pastagem 3 & 0 & 0 & 23 & 106 & 8 & 9 & 146 & 72,6 & 15,925 \\
\hline Pastagem 4 & 0 & 0 & 0 & 26 & 121 & 15 & 162 & 74,7 & 17,877 \\
\hline Área Urbana/solo exposto & 0 & 1 & 2 & 12 & 15 & 122 & 152 & 80,3 & 20,446 \\
\hline Total & 150 & 150 & 150 & 150 & 150 & 150 & 900 & & \\
\hline Exatidão do Produtor & 92,7 & 96,0 & 78,7 & 70,7 & 80,7 & 81,3 & & & \\
\hline \multicolumn{10}{|c|}{ Exatidão global $=83,3 ;$ Kappa $=0,80 ;$ Variância $=0,000222 ; Z$ calculado $=53,672$ e $Z$ tabelado $=1,96$} \\
\hline
\end{tabular}


que também teve $10 \%$ dos seus pixels assinalados erroneamente à classe pastagem 4 . A classe pastagem 2 (pastagem no nível de degradação moderado) que na classificação pelo Maxver apresentou baixa confusão com outras classes, teve confusão significativa com a classe pastagem 3, com 15,33\% dos pixels assinalados erroneamente a esta última classe.

Por outro lado, mata e café, respectivamente com 92,7 e 96\% dos pixels corretamente classificados, foram as classes que apresentaram os melhores resultados, conforme a Tabela 4 .

\section{Comparação entre a Rede Neural e o Maxver}

Um número considerável de trabalhos tem comparado a classificação de imagens de satélites por redes neurais com os métodos de classificação convencionais (Benediktsson et al., 1990; Bischof et al., 1992; Civco, 1993). De maneira geral, a utilização de redes neurais na classificação de dados de sensores remotos tem mostrado desempenho superior àquele obtido com o algoritmo Maxver. Segundo Kanellopoulos \& Wilkinson (1997), um aspecto que tem sido negligenciado nas comparações entre classificadores estatísticos e redes neurais, é a existência de diferenças significativas entre o desempenho desses classificadores, quando se consideram as classes, individualmente; então, para algumas classes a abordagem por redes neurais fornece maior exatidão, enquanto para outras os métodos convencionais são superiores. Este efeito resulta dos modelos matemáticos muito distintos assumidos nos diferentes classificadores e do modo como eles dividem o espaço característico.

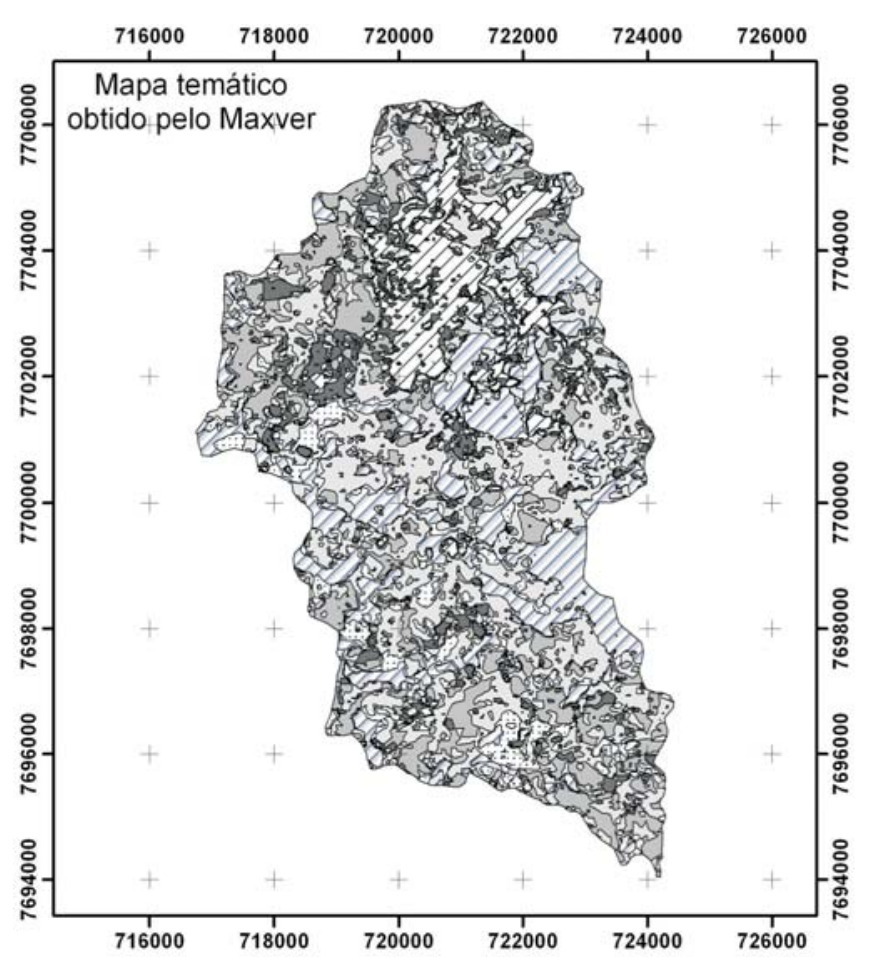

Dentre as classes avaliadas, a menor exatidão foi obtida para a classe pastagem 3 (pastagem no nível de degradação forte) para ambos os classificadores, com 59,3\% (Maxver) e 70,7\% (redes neurais) (Tabelas 2 e 4, respectivamente). Por outro lado, a maior exatidão individual foi obtida para a classe café, para a classificação realizada pelo algoritmo Maxver (100\%). Na classificação pela rede neural, a maior exatidão individual também foi obtida para a classe café, com $96 \%$ de exatidão (Tabela 4).

As classes avaliadas tiveram comportamento diferenciado entre os classificadores utilizados. Mata, café e pastagem 2, foram as classes mais bem classificadas pelo classificador Maxver, que produziu uma exatidão de 93,3, 100 e $94 \%$, respectivamente; por outro lado, as classes pastagem 3 , pastagem 4 e área urbana/solo exposto, foram mais bem identificadas pelo classificador por redes neurais, com 78,7, 70,7 e $81,3 \%$ de exatidão, respectivamente.

A análise da matriz de significância de Kappa (Tabela 5) indica não haver diferença significativa entre a classificação

Tabela 5. Matriz de significância de Kappa para os classificadores avaliados

\begin{tabular}{ccc}
\hline Imagens & Maxver & Rede Neural \\
Kappa & 0,793 & 0,800 \\
Variância & 0,000227 & 0,000222 \\
Maxver & 52,63 & - \\
Rede Neural & 0,330 & 53,69 \\
\hline
\end{tabular}

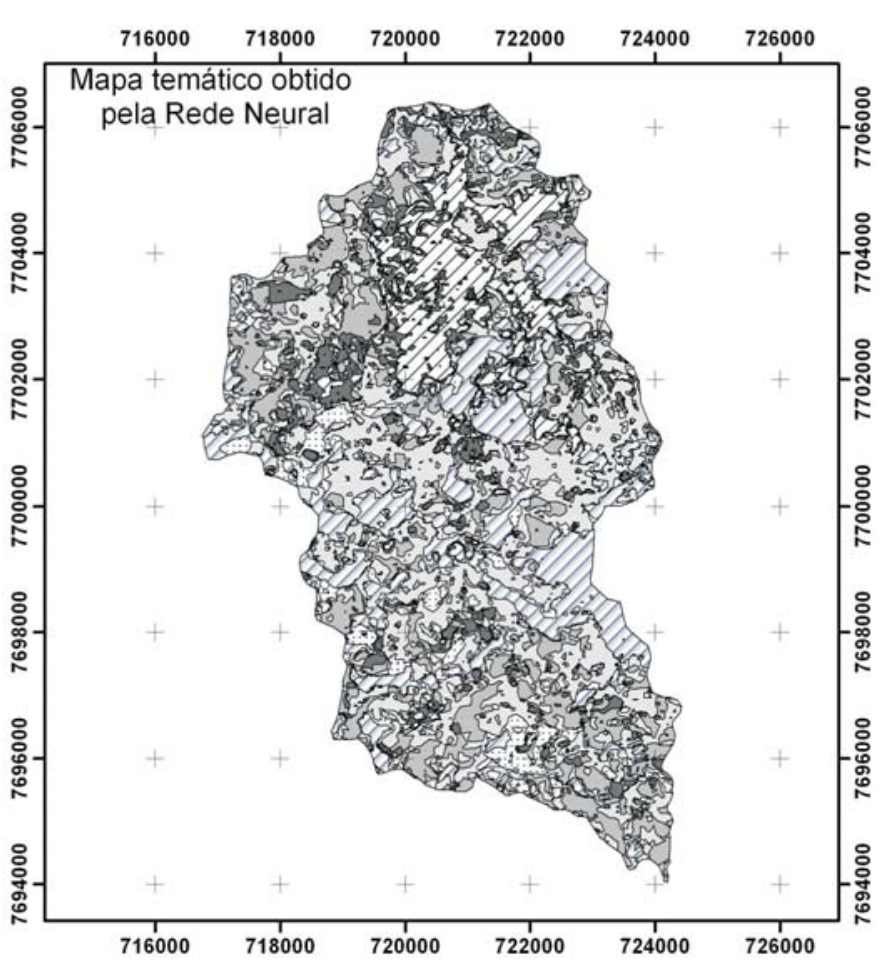

Legenda Mata Café
Pastagem 2
Pastagem 3
Pastagem 4

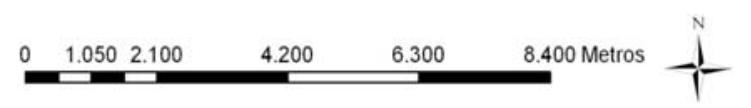

Figura 4. Mapas temáticos obtidos pelos classificadores 
por redes neurais e a classificação Maxver.

Os resultados obtidos indicam que, apesar das redes neurais apresentarem desempenho superior, em valores absolutos, para a classificação de dados de sensores remotos em relação ao algoritmo Maxver, os resultados (performances) não diferem estatisticamente para um nível de significância de 95\%. Embora os classificadores não tenham apresentado diferenças significativas $(0,80$ e 0,79 , respectivamente redes neurais e Maxver), há que se destacar que o classificador por redes neurais teve desempenho superior ao Maxver na classificação de duas das três classes de pastagem consideradas (pastagem 3 e pastagem 4), objetivo principal deste estudo.

Outrossim, há de se considerar que a abordagem por redes neurais requer mais tempo e mais recursos computacionais para treinar o sistema com as diferentes configurações de parâmetros testadas que a abordagem clássica pelo algoritmo Maxver; no entanto, para Yool (1998), a abordagem por redes neurais leva vantagem sobre os métodos convencionais de classificação supervisionada, como o algoritmo Maxver, porque estes se mostram inadequados e impraticáveis para o mapeamento de grandes áreas, o que não é o caso da área estudada.

O resultado da classificação final das imagens pelos classificadores testados é apresentado na Figura 4, na qual se verifica, visualmente, que a classificação pelo algoritmo Maxver e a classificação pela rede neural apresentam resultados muito próximos para todas as classes, com as discordâncias verificadas, basicamente, nos limites entre

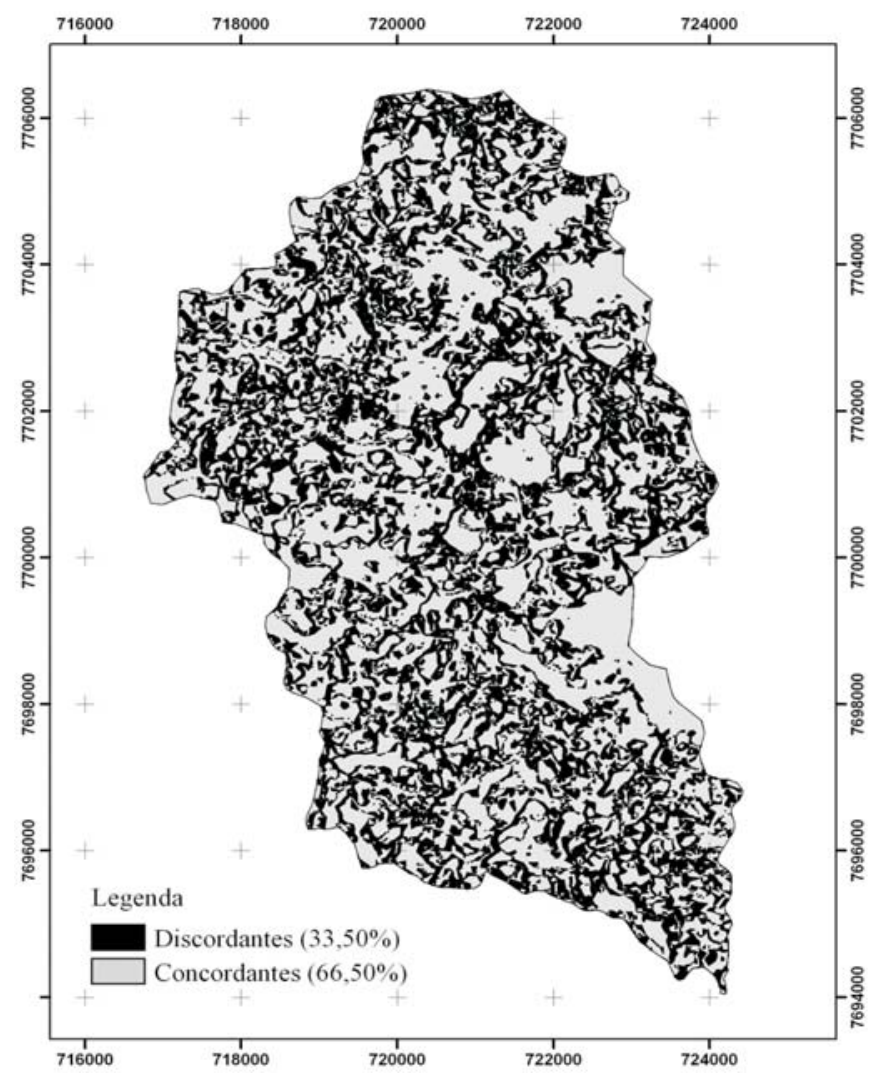

Figura 5. Comparação entre os mapas temáticos produzidos pelos classificadores as classes.

A análise da concordância entre as classificações mostra que a concordância entre a classificação pela rede neural e pelo algoritmo MAXVER foi de 66,50\% (Figura 5). No geral, os resultados alcançados pelos classificadores são semelhantes àqueles obtidos por Nascimento et al. (2006) que utilizaram a distância de Mahalanobis (formulações similares ao Maxver) para a classificação dos níveis de degradação em pastagens em uma microbacia na região de Viçosa, utilizando também uma imagem do sensor ASTER.

\section{CONCLUSÕES}

1. Os resultados alcançados neste estudo comprovam, mais uma vez, a eficiência da utilização de redes neurais artificiais para classificação do uso e cobertura do solo, podendo este classificador ser considerado uma alternativa viável para a classificação de níveis de degradação de pastagens, principalmente em grandes áreas, como no caso da Zona da Mata Mineira.

2. O classificador, baseado nas redes neurais, produziu uma exatidão na classificação geral em valores absolutos superior porém estatisticamente semelhante, comprovada através dos testes estatísticos utilizados, ao classificador clássico da máxima verossimilhança.

3. A exatidão geral da classificação baseada nas redes neurais, mostrou-se bastante influenciada pela arquitetura de rede utilizada.

4. Em todas as análises realizadas a classe que apresentou o maior erro para ambos os classificadores, foi a classe pastagem no nível de degradação forte, bastante confundida com a classe pastagem no nível de degradação muito forte.

\section{LITERATURA CITADA}

Atkinson, P. M.; Tatnall, A. R. L. Neural networks in remote sensing. International Journal of Remote Sensing, v.18, n.4, p.699709, 1997.

Benediktsson, J. A.; Swain, P. H.; Ersoy, O. K. Neural network approaches versus statistical methods in classification of multisource remote sensing data. Transactions on Geoscience and Remote Sensing, v.28, p.540-552, 1990.

Bischof, H.; Schneider, W.; Pinz, A. J. Multi-spectral classification of Landsat images using neural network. Transactions on Geoscience and Remote Sensing, v.30, p.482-490, 1992.

Bishop, Y.; Fienberg, Y. S.; Holland, P. Discrete multivariate analysis: Theory and practice. Cambridge: MIT Press. 1975. 557p.

Carvalho, M. M. Recuperação de pastagens degradadas em áreas de relevo acidentado. In: Dias, L. E.; Mello, J. W. V. (eds.). Recuperação de áreas degradadas. Viçosa: UFV-DPS/Sociedade Brasileira de Recuperação de Áreas Degradadas, 1998. p.149-161.

Cauldill, M. Neural network: Training tips and techniques. AI Experts, v.6, p.56-61, 1991. 
Civco, D. L. Artificial neural networks for land-cover classification and mapping. International Journal of Geographical Information System, v.7, n.2, p.173-186, 1993.

Congalton, R. G. A review of assessing the accuracy of classifications of remotely sensed data. Remote Sensing of Environment, v.49, n.12, p.1671-1678, 1991.

Congalton, R. G.; Mead, R. A. A review of discrete multivariate analysis techniques used in assessing the accuracy of remotely sensed data from error matrices. IEEE Transactions on Geoscience and Remote Sensing, v.24, p.169-174, 1986.

Foody, G. M.; Arora, M. K. An evaluation of some factors affecting the accuracy of classification by an artificial neural network. International Journal of Remote Sensing, v.18, n.4, p.799 - 810, 1997.

Hepner, G. F.; Logan, T.; Ritter, N.; Bryant, N. Artificial neural network classification using a minimal training set: comparison to conventional supervised classification. Photogrammetric Engineering and Remote Sensing, v.56, p.469-473, 1990.

Hirose, Y.; Yamashita, K.; Hijiya, S. Back-propagation algorithm which varies the number of hidden units. Neural Networks, v.4, p.61-66, 1991.

Kalkhan, M. A.; Reich, R. M.; Czaplewski, R. L. Variance estimates and confidence interval for Kappa measure of classification accuracy. Canadian Journal of Remote Sensing, v.23, n.3, p.210-216, 1997.

Kanellopoulos, I.; Varfis, A.; Wilkinson, G. G.; Mégier, J. Landcover discrimination in SPOT HRV imagery using an artificial neural network: A 20-class experiment. International Journal of Remote Sensing, v.13, p.917- 924, 1992.

Kanellopoulos, I.; Wilkinson, G. G. Strategies and best practice for neural network image classification. International Journal of Remote Sensing, v.18, n.4, p.711-725, 1997.

Master, T. Practical neural network recipes in C++. San Diego: Academic, 1993. 493p.

Monserud, R. A.; Leemans, R. Comparing global vegetation maps with the Kappa statistic. Ecological Modelling, v.62, p.275293, 1992.

Moreira, L.; Assad, E. D. Segmentação e classificação supervisionada para identificar pastagens degradadas. Workshop Brasileiro de Geoinformática, 2, 2000, São Paulo. Anais... São Paulo: Sociedade Brasileira de Computação, 2000. 15p.

Nascimento, M. C.; Riva, R. D. D.; Chagas, C. S.; Oliveira, H.; Dias, L. E.; Fernandes Filho, E. I.; Soares, V. P. Uso de imagens do sensor ASTER na identificação de níveis de degradação em pastagens. Revista Brasileira de Engenharia Agrícola e Ambiental, v.10, p.196-202, 2006.
Paola, J. D.; Schowengerdt, R. A. Comparisons of neural networks to standard techniques for image classification and correlation. Proceedings of the International Geoscience and Remote Sensing Symposium IGARSS’94, Pasadena, 1994, Pasadena: Institute of Electrical and Electronics Engineers, Piscataway, p.1404-1406. 1994.

Sano, E. E.; Chaves, J. M.; Bezerra, H. S.; Feitoza, L. Identificação dos principais tipos de pastagens cultivadas do Cerrado a partir de Sensoriamento Remoto. International Symposium: Soil Functioning under Pastures in Intertropical Areas, 2000, Brasília. Anais... Brasília: Embrapa Cerrados - IRD, 2000. CD-Rom.

Skidmore, A. K.; Bijker, W.; Schmidt, K.; Kumar, L. Use of remote sensing and GIS for sustainable land management. ITC Journal, v.3/4, p.302-315, 1997.

Skidmore, A.; Turner, B. Assessing the accuracy of resource inventory maps. Proceedings for Global Natural Resource Monitoring and Assessments: Preparing for the 21 ${ }^{\text {th }}$ Century. Venice, v.2, p.524-535, 1989.

Spain, J. M.; Gualdrón, R. Degradación e rehabilitación de pasturas. In: Lascano, C.; Spain, J. M. (eds.). Establecimiento y renovación de pasturas. Cali: CIAT, 1991. 426p.

Story, M.; Congalton, R. G. Accuracy assessment: A user's perspective. Photogrammetric Engineering and Remote Sensing, v.61, p.391-401, 1986.

Venkatesh, Y. V.; Raja, S. On the classification of multispectral satellite images using the multilayer perceptron. Pattern Recognition, v.36, p.2161 - 2175, 2003.

Vieira, C. A. O. Accuracy of remotely sensing classification of agricultural crops: A comparative study. Nottingham: University of Nottingham, 2000. 327p. PhD Thesis

Vilela, M. Uso de diferentes métodos de retificação geométrica e classificação digital de uma imagem TM/LANDSAT - 5. Viçosa: UFV, 1998. 118p. Dissertação Mestrado

Yool, S. R. Land cover classification in rugged areas using simulated moderate-resolution remote sensor data and an artificial neural network. International Journal of Remote Sensing, v.19, p.85-96, 1998.

Zell, A.; Mamier, G.; Vogt, M.; Mache, N.; Hübner, R.; Döring, S.; Herrmann, K.; Soyez, T.; Schmalzl, M.; Sommer, T.; Hatzigeorgiou, A.; Posselt, D.; Schreiner, T.; Kett, B.; Clemente, G.; Wieland, J.; Gatter, J. Stuttgart Neural Network Simulator v4.2. Stuttgart: University of Stuttgart/Institute for Parallel and Distributed High Performance Systems/University of Tübingen - Wilhelm-Schickard-Institute for Computer Science/Department of Computer Architecture. 1996. 388p. 